\title{
Integrated optimization of urban agglomeration passenger transport hub location and network design
}

\author{
Bofeng Fan ${ }^{1,2^{*}}$, Yuling Yang ${ }^{2}$ and Liang $\mathrm{Li}^{3}$
}

\begin{abstract}
Because of the influence of comprehensive hub construction on network flow, this paper adopts the bi-level programming model (BPM) to solve the problem about the integrated optimization of multi-hub location and network layout. The upper level model is designed to minimize the network operating cost, network transformation cost, and hub construction cost; the lower level is the user equilibrium distribution model at fixed demands. Besides, this paper designs the improved genetic algorithm to solve the bi-level programming model and validates the model validity and algorithm feasibility through the computing example. The model has a high application value for solving the problem of comprehensive optimization of intercity passenger transport hub location and intercity passenger transport network design in the process of urban agglomeration development.
\end{abstract}

Keywords: Integrated optimization, Improved genetic algorithm, Passenger transport hub location, Traffic network design, Urban agglomeration

\section{Introduction}

Passenger transport hub, as the one key node of intercity passenger transport network, is the important place for passenger gathering, distributing ETC in transit. Besides, the transport location advantage of passenger hub has an obvious positive effect on the population, industry accumulation, and resource allocation. The rational distribution of hub and network cannot only improve the transport system function in urban agglomeration and transport efficiency but also promote the harmonious development of urban regional economy significantly.

\subsection{Methods/experimental}

The issues of transportation network design have generally divided into discrete network design problems (DNDP), continuous network design questions (CNDP), and mixed network design problems (MNDP) according to the types of decision variables. For the urban intercity passenger transport network, which is generally composed of high-grade

\footnotetext{
* Correspondence: fbfyfbf@hotmail.com

${ }^{1}$ School of Transportation and Logistics, Southwest Jiaotong University, Chengdu 610031, China

${ }^{2}$ School of Environment and Resources, Southwest University of Science and Technology, Mianyang 621010, China

Full list of author information is available at the end of the article
}

highways, intercity railways, or high-speed rails, the intercity rapid passenger transport network is in the process of rapid development, and mostly, new lines update the network. This paper adopts a discrete network design model. To simulate the rapid growth of line construction requirements, a bi-level planning method has adopted to establish a model for simultaneous optimization of multi-hub site selection and network layout. The upper model aims at minimizing the total cost of network operation and construction. The lower model is a user-balanced assignment model with fixed requirements. This paper uses MATLAB software to design an improved genetic algorithm to solve the bi-level planning model, considering the impact of the integrated hub construction on network traffic. We make comprehensive decisions on the layout of the line and the layout of the hub.

\section{Literature review}

Transportation hub location and transportation network design are the main subjects of transportation planning, for which the scholars have made many studies: in terms of hub location optimization, Ebery established the mixed-integer programming model and offered the related 
algorithm to solve the multi-hub location issue with capacity limitation [1]. Topcuoglu has studied non-capacity single hub selection mode and then designed the genetic algorithm accordingly [2]. Liu qiang et al. have based on network stochastic traffic distribution [3], and Zhang Bing et al. have adopted the niche genetic annealing algorithm to solve the optimization of network multi-hub location [4]. Li Daikun et al. have targeted at minimization of total demands, took the hub coverage area into consideration, and adopted the adaptive immune clone algorithm to solve the layout optimization of regional urban nodes [5]; Li Tingting et al. built the optimization model of passenger transport hub hierarchical layout [6]. These studies above mainly proposed the model and algorithm of multi-hub location optimization, but without considering the influence of hub construction on network. Once the hub is built, the rapid development of hub city would increase certain amount of traffic demands, the hub function can change the network transportation mode, and then, the network flow distribution accordingly. Hence, it is to fulfill the harmonious and high-efficient network operation in the transportation network of urban agglomeration, the hub and network should be integrated. Liu Canqi has studied the integration of hub selection and continuous network optimization and applied the iterative optimal algorithm [7]. Sun Yang constructed a model and applied the immune clone algorithm to solve the integrated optimization issue of multi-hub location and mixed network design [8]. Therefore, this paper, based on the existing researches on the hub planning and network design problem, studies the integrated configuration model of intercity transport hub and network in urban agglomeration.

\section{Model construction}

\subsection{Model symbol definition}

The related symbols in the model are defined as follows. $N$ is the number of nodes in transportation network; $M$ is the number of hubs to be built; $A_{1}$ is the sum of existing roads or railway sections (section) in intercity transportation network; $A_{2}$ is the sum of sections to be added in the network; $A$ is the sum of all sections in the network, and $A=A_{1} \cup A_{2} ; R$ is the sum of origin in the transportation network $r \in R ; S$ is the sum of destination, $s \in S ; x_{a}$ is the traffic flow of any section $a ; t_{a}(x)$ is the travel time of section $a$, as function of link flow; $K_{r s}$ is the path set of OD pair: $r$ and $s ; f_{k}^{r s}$ is the flow of the path $k$ between $r$ and $s ; q_{r s}$ is the traffic volume between $r$ and $s ; c_{a}$ is the traffic capacity of section $a ; g_{a}\left(y_{a}\right)$ means cost of new line $a$. $\lambda$ is the unit conversion factor between time and currency value; $y_{a}$ is the decision variable of the alternative section $a, y_{a} \in\{0,1\} ; b_{m n}$ is the construction cost of the hub; $z_{m n}$ is the decision variable of hub location, and when the hub $m$ is located at $n$, it is 1 , otherwise $0 ; \delta_{a, k}^{r s}$ is the decision variable related to $a$ and $k$, and when $a$ is located in the path $k$ between $r$ and $s, \delta_{a, k}^{r s}$ is 1 , otherwise 0 .

\subsection{Bi-level programming model}

Based on the existing research findings, this paper adopts the administrator-dominant and user-following bi-level mathematical programming model to describe the problem. This bi-level programming model consists of upper level and lower level: the upper level is for the complicated optimization problem, including optimal decision variable of lower level, while the lower level is programming problem with decision variable parameter of the upper level.

To make comprehensive decision of urban intercity passenger hub and network, the transportation operation department would consider the network operation efficiency to ensure smooth network operation, and then, in the model, the minimum of total system impedance in the transportation network is applied to describe this demand. The traffic infrastructure construction and capital management department also prefer the minimization of total investment, including costs of road reconstruction and expansion, new-built lines, and hub construction. Therefore, the upper level in this paper is designed as a network and hub programming plan, to be the multi-objective optimization with network operation cost (total network impedance) and network transformation cost. The lower level is to solve the traffic distribution problems, which can be divided into determined traffic distribution problem and stochastic traffic distribution problem according to the users' knowledge about the trip network information. Generally, the determined distribution model is adopted for programming level, so this paper applies the user equilibrium traffic distribution model (determining demands) as the representation of the lower level model in the optimization model of this paper.

Upper level model $(U)$ :

$$
\begin{aligned}
\min C= & \lambda \sum_{a \in A_{1}} x_{a} t_{a}\left(x_{a}\right)+\lambda \sum_{a \in A_{2}} x_{a} t_{a}\left(x_{a}, y_{a}\right) \\
& +\sum_{a \in A_{2}} g_{a}\left(y_{a}\right)+\sum_{n \in N} \sum_{m \in M} b_{m n} z_{m n}
\end{aligned}
$$

$$
\begin{aligned}
& \text { s.t. } \quad \sum_{m \in M} z_{m \mathrm{n}}=1, \quad \forall n \in N \\
& \sum_{n \in N} z_{m n}=1, \quad \forall m \in M \\
& y_{a}=\{0,1\}, \quad \forall a \in A_{2}
\end{aligned}
$$

Lower level model $(L)$ : 


$$
\begin{aligned}
& \min T=\sum_{a \in A_{1}} \int_{0}^{x_{a}} t_{a}(x) d x+\sum_{a \in A_{2}} \int_{0}^{x_{a}} t_{a}\left(x, y_{a}\right) d x \\
& \text { s.t. } \quad \sum_{k \in K} \int_{k}^{r s}=q_{r s}+\sum_{\forall r \in R, s \in S,}\left(d_{m r} p_{m r}, d_{m s} p_{m s}\right), \\
& x_{a}=\sum_{r} \sum_{s} \sum_{k} f_{k}^{r s} \cdot \delta_{a, k}^{r s}, \quad \forall a \in A, \quad r \in R, \quad s \in S, \quad k \in K, \\
& f_{k}^{r s} \geq 0, \quad \forall r \in R, \quad s \in S, \quad k \in K
\end{aligned}
$$

Where the objective function (1) is the network and hub construction cost, (2) means only one hub can be built for each node, (3) means that every hub must be selected and built on one node, (4) is the range constraint of new-built lines, (6) is the traffic flow between any two travel points of the built hub, and in (6), $d_{m r}$ and $d_{m s}$ are the decision variable, and when hub $m$ is located at $r$ and $s$, it is 1 , otherwise 0 .

For the link impedance function in the model, the travel time formula proposed by Bureau of Public Road (BPR) is adopted as follows.

$$
t_{a}=t_{a}^{0}\left[1+\alpha\left(\frac{x_{a}}{c_{a}}\right)^{\beta}\right]
$$

where $c_{a}$ is the traffic capacity of $a ; t_{a}^{0}$ is the free travel time of section $a ; \alpha$ and $\beta$ are the retardation coefficients, and the specific values can be obtained by regression analysis for actual data.

\subsection{Solving algorithm}

Firstly, the upper level programming administrator determined the hub and new-built lines, made traffic distribution at lower level, then returned back to solve the cost at the higher level, and finally repeated so to acquire the optimal solution. For the holistic methods of lower programming, the stochastic equilibrium traffic distribution has adopted, but the genetic algorithm was applied for iterative calculation to make optimization. The key items are described as follows.

\subsubsection{Antibody coding}

In the upper level programming model, population coding was adopted for antibody. Based on the decision variable, set two antibodies $\mathrm{A}$ and $\mathrm{B}$.

$A=\left[a_{1}, a_{2}, \ldots, a_{i}\right], i \in n, n$ is the number of total nodes, where $a_{i}$ is the numbering of built nodes, and real-number encoding is used for node selection, to select the node numbering according to the reservation number. The antibody $B=\left[b_{1}, b_{2}, \ldots, b_{i}\right], i \in n, n$ is the

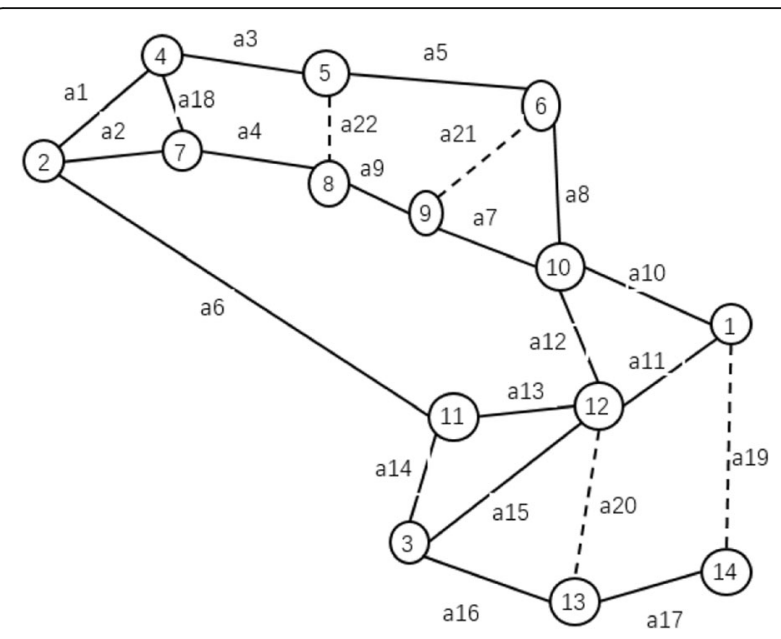

Fig. 1 High way network status in example

total of roads to be built, and $b_{i}$ coded in the binary coding, is the road to be built; if it needs to be built, the value is 1 , otherwise 0 .

In the lower programming model, the antibody encoding includes $n$ antibody, and the number of antibody equals to that of OD pairs. $X=\left[x_{1}, x_{2}, \ldots, x_{n}\right], x_{n}=\left[p_{1}\right.$, path $\left._{1} ; p_{2}, \operatorname{path}_{2} ; \ldots ; p_{i}, \operatorname{path}_{i}\right]$, where $i$ is the number of times for OD pair incremental traffic distribution, $p$ is the flow, and path means the path, traffic distribution flow, traffic distribution path (the total flow is divided into $n$ groups, and $n$ is generated randomly at different flows, then it is allocated to the shortest path) for $n$ times of traffic distribution randomly for corresponding OD pair, dynamic length, and real-number encoding.

\subsubsection{Elitism selection and cataclysm}

Our algorithm integrates the elitism and cataclysm. The elitism selection is, by duplicating the optimal solution of one generation to the next generation, to prevent the optimal solution generated in the evolving process from crossover and mutation damage. Cataclysm means that, by killing all or some outstanding individuals at the convergence of algorithm, the individuals far from the current extremal can have the chance to be evolved more sufficiently; this improvement can ensure the genetic algorithm to be jumped out of local extremum, when this algorithm falls in the local optimal. For these two seemingly contradictory improving strategies, this algorithm fulfills the integration of cataclysm and elitism selection in the following way. When a new generation is created at each iteration cycle, the most outstanding individual should be duplicated to the next generation unconditionally. Nevertheless, if in the continuous $N$ generations, there is no more outstanding individual or lower change of population diversity, the algorithm easily falls into local optimal, so the cataclysm strategy can be applied to kill one or more optimal individuals 


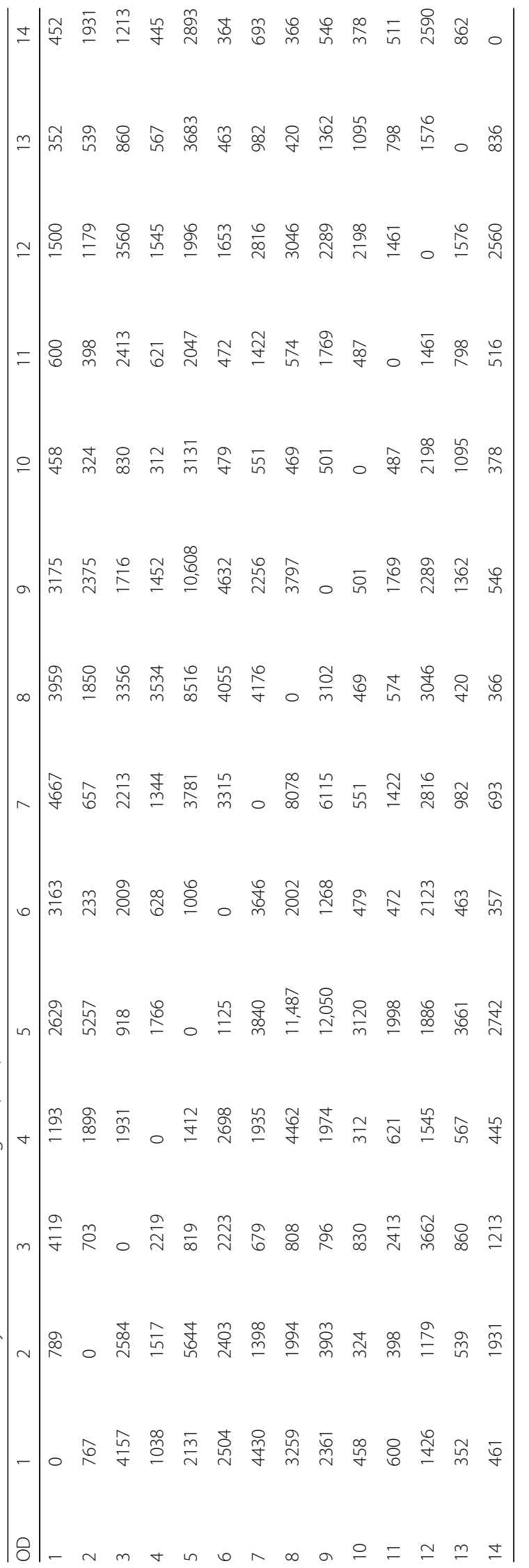


of the population in the next one or multi-iterations and then help jump out of local extremum. The elitism strategy is followed with each generation, while the cataclysm strategy can be applied every several generations, to fulfill the integration of both strategies.

\subsubsection{Adaptive crossover, mutation, and cataclysm probability}

Adaptive crossover operator was used in the algorithm design, to ensure taking the evolution direction beneficial to algorithm convergence, by adjusting crossover probability according to the fitness function of individual in every generation. In the basic genetic algorithm, to select the individual by setting constant crossover probability and to make crossover operation by selecting crossover points randomly (roulette), it might lead to offspring individual inferior to parental individual, which should be avoided in our algorithm.

(1) Crossover operator

$$
p_{i}=\left(f_{\max }-f_{i}\right) /\left(f_{\max }-f_{\min }\right)
$$

where $i$ is individual, $f_{i}$ is fitness of individual, $p_{i}$ is crossover probability, $f_{\max }$ and $f_{\min }$ are the max and min, respectively, of population fitness.

(2) Crossover, mutation, and cataclysm probability

$$
P(t)=p_{0} *\left(1+\frac{t}{\operatorname{maxGen}}\right)
$$

$P(t)$ is the corresponding probability of each iteration cycle, $p_{0}$ is initial probability (different numbers for crossover, mutation, and cataclysm), $t$ is current iteration cycle, and maxGen is max iteration cycle.

\subsubsection{Algorithm flow}

Step 1: Define initial network value;

Step 2: Initialize the antibody population; set the antibody population size, maximum iteration time, stopping criterion, and calculate the fitness of every individual in the initial antibody population;

Step 3: Based on the given antibody population size, make cloning for every antibody (individual);
Step 4: Based on crossover probability and objective function value, make crossover operation for cloned antibody (individual);

Step 5: According to mutation probability, make mutation for cloned antibody (individual);

Step 6: According to cataclysm probability, make cataclysm for cloned antibody (individual);

Step 7: Make the clone (individual) selection, to select new antibody (individual) population per the selection rule;

Step 8: Judge whether meeting the stopping criteria; if yes, stops the calculation and output the final plan; if not, go back to Step 3 .

\section{Model validation}

\subsection{Example design}

This paper took the highway network in urban agglomeration of one region, with the regional prefecture city as the network node. The simplified road network is shown in Fig. 1, including 14 nodes, 22 road sections (18 are built lines, and 4 alternative lines); a1-a18 are the existing built road sections, and a19-a22 are alternative ones.

Tables 1 and 2 show OD demand matrix; Table 3 is information of road sections. Suppose the construction cost of single hub is 0.2 billion yuan, $\lambda$ is defined according to actual condition, and the impedance function coefficient is $\alpha=0.15, \beta=4$; the cities 1,2 , and 3 are fixed hubs, and now it is required to select two more cities from other node cities as the comprehensive hub; so it is necessary to determine the hub location and whether the alternative road sections are built.

Based on the programming model above, this paper applies the improved genetic algorithm and MATLAB programming. As shown in Fig. 2, the parameters have given to calculate the selected nodes: 8 and 12 , and the new-built road a19.

Optimization results and fitness curve are shown in Fig. 2 and 3, respectively. The program shows the optimal solution at 26th iteration, with better convergence.

\subsection{Results and analysis}

4.2.1 Calculation results

Combined with the above bi-level planning model and examples, this paper uses MATLAB software to write an improved genetic algorithm to get the solution; we can set the antibody population size 20 , the maximum number of iterations is 50 . The initial crossover probability, mutation

Table 2 Absorbed traffic flow (PCU)

\begin{tabular}{lllllllllllllll}
\hline OD & 1 & 2 & 3 & 4 & 5 & 6 & 7 & 8 & 9 & 10 & 11 & 12 & 13 & 14 \\
\hline Hub 1 & 103 & 150 & 205 & 77 & 174 & 87 & 138 & 326 & 152 & 48 & 59 & 164 & 71 & 45 \\
Hub 2 & 387 & 260 & 43 & 166 & 88 & 176 & 201 & 287 & 155 & 74 & 217 & 306 & 81 & 118 \\
\hline
\end{tabular}


probability, and catastrophe probability are set to $0.1,0.2$, and 0.1 , respectively, the value of $\lambda$ is 0.0001 . The calculation results are shown in Fig. 2, and fitness curve is shown in Fig. 3. In addition to the established hubs, node 8 and node 12 have selected as new hubs. At the same time, the new highway has selected as a19.

\subsubsection{Analysis of results}

(1) It can be seen from the fitness curve that as the number of iterations increases, the affinity of the antigen and antibody increases, and the optimal fitness value and the average fitness value both tend to decrease. The results show that the entire antibody population is continuous. The optimal solution converges to verify the feasibility of the algorithm. At the same time, Fig. 3 shows that the program achieves an optimal solution when it is iterating 26 times, and the convergence is good. The results show that this algorithm uses the combination of elite selection and catastrophe methods and the setting of

Table 3 Road section information in road network

\begin{tabular}{|c|c|c|c|c|c|}
\hline $\begin{array}{l}\text { Line } \\
\text { no. }\end{array}$ & $\begin{array}{l}\text { Line length } \\
(\mathrm{km})\end{array}$ & $\begin{array}{l}\text { Speed } \\
(\mathrm{km} / \mathrm{h})\end{array}$ & $\begin{array}{l}\text { Zero-flow } \\
\text { impedance } \\
\text { (min) }\end{array}$ & $\begin{array}{l}\text { Traffic } \\
\text { capacity } \\
\text { (PCU/h) }\end{array}$ & $\begin{array}{l}\text { New-built line } \\
\text { cost (million } \\
\text { yuan) }\end{array}$ \\
\hline 1 & 101 & 100 & 61 & 2000 & \\
\hline 2 & 94 & 100 & 56 & 2000 & \\
\hline 3 & 64 & 100 & 38 & 2000 & \\
\hline 4 & 76 & 100 & 46 & 2000 & \\
\hline 5 & 81 & 100 & 49 & 2000 & \\
\hline 6 & 168 & 100 & 101 & 2000 & \\
\hline 7 & 69 & 100 & 41 & 2000 & \\
\hline 8 & 54 & 100 & 32 & 2000 & \\
\hline 9 & 32 & 100 & 19 & 2000 & \\
\hline 10 & 93 & 100 & 56 & 2000 & \\
\hline 11 & 116 & 100 & 70 & 2000 & \\
\hline 12 & 96 & 100 & 58 & 2000 & \\
\hline 13 & 50 & 100 & 30 & 2000 & \\
\hline 14 & 98 & 100 & 59 & 2000 & \\
\hline 15 & 98 & 100 & 59 & 2000 & \\
\hline 16 & 83 & 100 & 50 & 2000 & \\
\hline 17 & 62 & 100 & 37 & 2000 & \\
\hline 18 & 34 & 100 & 20 & 2000 & \\
\hline 19 & 213 & 100 & 128 & 0 & 2130 \\
\hline 20 & 108 & 100 & 65 & 0 & 1080 \\
\hline 21 & 125 & 100 & 75 & 0 & 1250 \\
\hline 22 & 109 & 100 & 65 & 0 & 1090 \\
\hline
\end{tabular}

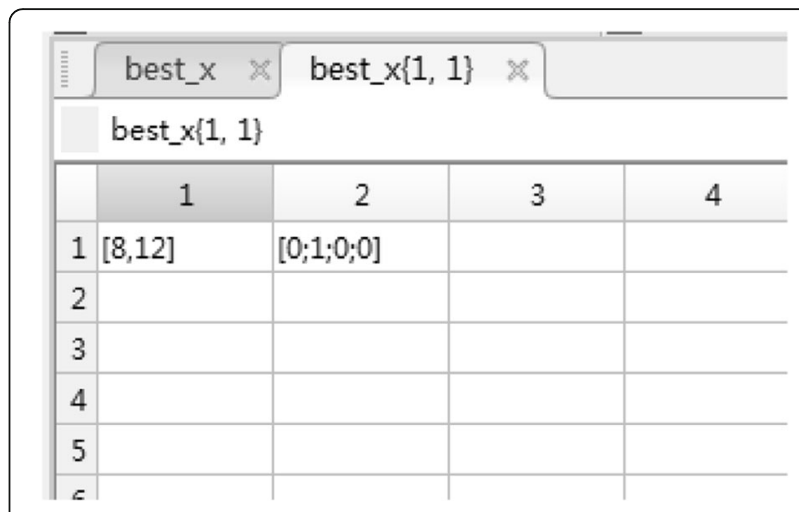

Fig. 2 Optimization results

adaptive crossover operator to enhance the effectiveness of the algorithm.

(2) Different from the commonly used Frank-Wolf assignment algorithm, this paper uses a genetic algorithm to optimize the stochastic equilibrium flow assignment iteratively. In this way, the upper and lower layer models are all iteratively optimized using the genetic algorithm, and the programming is easier under the same conditions using MATLAB software. Its adaptability is stronger.

(3) Due to limited data, the models and algorithms involved in this paper need to combine with actual planning cases to further verify and improve. At the same time, we can also try other ways to improve the efficiency of calculations further for the method of lower level distribution and the selection of programming languages.

\section{Conclusions}

The practice indicates the following: the expansion of intercity passenger transportation network in urban

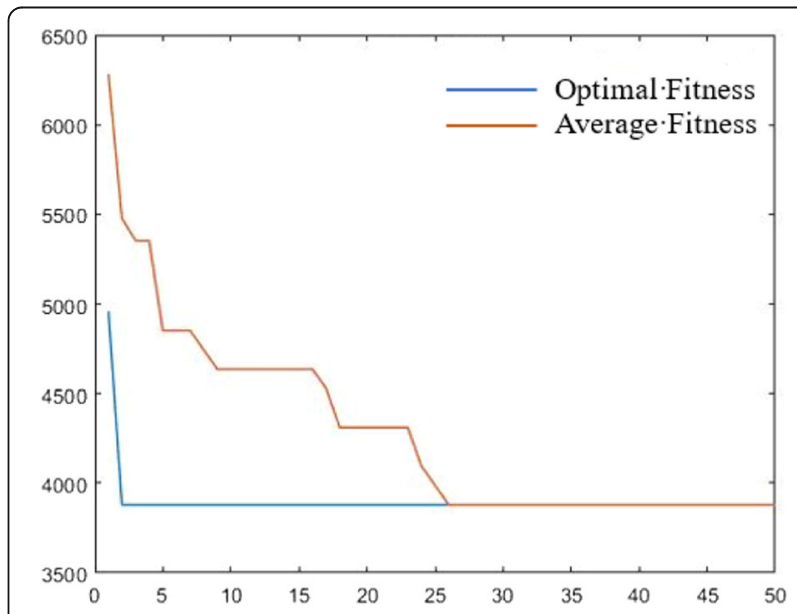

Fig. 3 Fitness curve 
agglomeration can attract more and more intercity passenger flow, and comprehensive passenger transport hub construction is the inevitable demand. The passenger transport hub construction will certainly attract new travel demands, producing a greater influence on network layout. In the network planning of rapidly developing intercity transportation, it is important to take both the hub location and network layout into consideration.

Because of the current situation of line planning one by one in intercity passenger transport network development, disjunction of comprehensive hub location, and network layout, this paper adopts the bi-level programming model for simultaneous optimization of both discrete network design and hub selection, designs the improved genetic algorithm to optimize the cost objective at the upper level and network traffic distribution at lower level. The example above shows our model, and algorithm is of great practicality and efficiency, which can be a feasible method to solve the simultaneous optimization of intercity transport multi-hub selection and network layout.

The future research directions are as follows: further study on the prediction method of the traffic volume and the transfer traffic volume induced by the new passenger transport hub, design other algorithms of this model and compare them, research on the development and application of this model for a comprehensive traffic network composed of various traffic modes.

\section{Abbreviations}

BPM: Bi-level programming model; CNDP: Continuous network design questions; DNDP: Discrete network design problems; MNDP: Mixed network design problems

\section{Acknowledgements}

The research presented in this paper was supported by the School of Transportation and Logistics, Southwest Jiaotong University, Chengdu 610031, China; School of Environment and Resource, Southwest University of Science and Technology, Mianyang 621010, China; School of Economics and Management, Southwest Jiaotong University, Chengdu 610031, China.

\section{Availability of data and materials}

Data sharing not applicable to this article as no datasets were generated or analyzed during the current study.

\section{Authors' contributions \\ $\mathrm{BF}$ is the main author of this paper who puts forward the main method, designs the model, and uses the improved genetic algorithm to verify the example. YY provides effective help to the feasibility and efficiency of the model algorithm. LL puts forward some important suggestions for the establishment of the model. All the authors read and approved the final manuscript.}

\section{Authors' information}

Bofeng Fan is pursuing his Ph.D. degree in Department of Railway Transportation Engineering, School of Transportation and Logistics, Southwest Jiaotong University (SWJTU). He is currently a lecturer in the Department of Traffic Engineering, Southwest University of Science and Technology, China. His research interests include comprehensive transportation and traffic planning.

Yuling Yang received her M.S. degree in the Department of Traffic

Engineering, School of Transportation and Logistics, Southwest Jiaotong
University (SWJTU). She is currently a lecturer in the Department of Traffic Engineering, Southwest University of Science and Technology, China. Her research interests include logistics system planning, complex network. Liang Li received his Ph.D. degree in the School of Economics and Management (SEM), Southwest Jiaotong University. He is now an associate professor in the School of Economics and Management, Southwest Jiaotong University, China. His research interests include ethical decision making behaviors and optimal allocation of resources.

\section{Competing interests}

The authors declare that they have no competing interests.

\section{Publisher's Note}

Springer Nature remains neutral with regard to jurisdictional claims in published maps and institutional affiliations.

\section{Author details}

${ }^{1}$ School of Transportation and Logistics, Southwest Jiaotong University, Chengdu 610031, China. ${ }^{2}$ School of Environment and Resources, Southwest University of Science and Technology, Mianyang 621010, China. ${ }^{3}$ School of Economics and Management, Southwest Jiaotong University, Chengdu 610031, China.

Received: 14 May 2018 Accepted: 18 June 2018

Published online: 04 July 2018

References

1. J Ebery, M Krishnamoorthy, A Ernst, The capacitated multiple allocation hub location problem: formulations and algorithms. Eur. J. Oper. Res. 120(3), 614-631 (2000)

2. H Topcuoglu, F Corut, M Ermis, Solving the uncapacitated hub location problem using genetic algorithms. Comput. Oper. Res. 32(4), 967-984 (2005)

3. Q Liu, HP Lu, QY Wang, Bi-level programming model for regional integrated transportation hub layout. J.Southeast Univ.: Nat. Sci.Ed. 40(6), 1358-1363 (2010)

4. B Zhang, W Deng, Location model of transport junction in economic circle and its algorithm. J. Transp. Syst. Eng. Inf. Technol. 10(4), 1358-1363 (2010)

5. DK Li, SW He, YS Shen, Layout optimization of comprehensive transportation hub based on immune clone algorithm. J. Wuhan Univ. Techno.: Transpor. Sci. Eng. 36(2), 382-386 (2012)

6. TT Li, R Song, SW He, HD Li, Regional passenger hub layout optimization based on hierarchical location model. J. Transp. Syst. Eng. Inf. Technol. 14(6), 37-41 (2014)

7. CQ Liu, Model of simultaneously optimizing the transport pivot siting and network design and its algorithm. J. Highway .Transport. Res. Dev. 20(3), 113-116 (2003)

8. Y Sun, R Song, SW He, HB Wang, Integrated optimization of multiple hubs location and mixed network design based on immune clone algorithm. J. Highway .Transport. Res. Dev. 26(12), 102-106 (2009)

\section{Submit your manuscript to a SpringerOpen ${ }^{\mathcal{O}}$ journal and benefit from:}

- Convenient online submission

- Rigorous peer review

- Open access: articles freely available online

- High visibility within the field

- Retaining the copyright to your article

Submit your next manuscript at $>$ springeropen.com 